\title{
Mastoiditis aguda con complicación intracraneal. Reporte de un caso pediátrico
} Acute mastoiditis with intracranial complication in a pediatric patient

\author{
Lic. Sara Laliena Aznara, Lic. Cayetana Verástegui Martínez $z^{b}$ Lic. Raquel Bernadó Fonz ${ }^{a}$, \\ Lic. Irene Baquedano Lobera ${ }^{a}$ y Lic. Matilde Bustillo Alonso ${ }^{c}$
}

\section{RESUMEN}

La mastoiditis aguda es una infección de las celdillas mastoideas, generalmente, secundaria a la progresión de una otitis media aguda. Las bacterias aisladas con más frecuencia en las mastoiditis son Streptococcus pneumoniae, Streptococcus pyogenes y Staphylococcus aureus. La infección mastoidea puede extenderse por contigüidad, afectar a estructuras vecinas y dar lugar a complicaciones intra- o extracraneales. Las más frecuentes son las intracraneales, entre las que se incluyen la meningitis, el absceso cerebeloso o del lóbulo temporal, el absceso epi- o subdural y la trombosis de senos venosos. Se presenta el caso de una niña de 4 años que desarrolló dos complicaciones intracraneales (absceso epidural y trombosis de senos venosos transverso y sigmoideo) a partir de una mastoiditis aguda producida por Streptococus pyogenes.

Palabras clave: mastoiditis, absceso epidural, trombosis de los senos intracraneales, pediatría.

\begin{abstract}
Acute mastoiditis is an infection that affects the mastoid air-cell system, usually due to the progression of an acute otitis media. The bacteria most frequently isolated in acute mastoiditis are Streptococcus pneumoniae, Streptococcus pyogenes and Staphylococcus aureus. The mastoid infection can extend affecting contiguous structures and producing intra or extracranial complications. The most frequent ones are intracranial complications, including meningitis, temporal lobe or cerebellar abscess, epidural or subdural abscess and venous sinus thrombosis.

We present the case of a 4-year-old girl who developed two intracranial complications (intracranial epidural abscess and transverse and sigmoid sinus thrombosis) initiated in an acute mastoiditis produced by Streptococcus pyogenes.

Key words: mastoiditis, epidural abscess, intracranial sinus thrombosis, pediatrics.
\end{abstract}

http: / / dx.doi.org/10.5546/ aap.2020.e166

a. Servicio de Pediatría, Hospital Infantil Miguel Servet, Zaragoza, España.

b. Servicio de Pediatría, Hospital Ernest Lluch, Calatayud.

c. Unidad de Infectología Pediátrica, Hospital Infantil

Miguel Servet, Zaragoza, España.

Correspondencia:

Lic. Sara Laliena Aznar: saralaliena@gmail.com

Financiamiento: Ninguno.

Conflicto de intereses: Ninguno que declarar.

Recibido: 14-1-2019

Aceptado: 16-9-2019
Cómo citar: Laliena Aznar S, Verástegui Martínez C, Bernadó Fonz R, Baquedano Lobera I, Bustillo Alonso M. Mastoiditis aguda con complicación intracraneal. Reporte de un caso pediátrico. Arch Argent Pediatr 2020;118(2):e166-e169.

\section{INTRODUCCIÓN}

La mastoiditis aguda (MA) aparece como resultado de la extensión de una otitis media aguda (OMA) y provoca la infección de las celdillas mastoideas del hueso temporal. Se trata de la complicación más frecuente de una OMA.

La MA es una enfermedad potencialmente grave que debe sospecharse ante la presencia de celulitis en la región mastoidea, junto con el despegamiento del pabellón auricular. Tras la inflamación inicial de la mastoides, esta puede evolucionar hacia la destrucción de las celdillas óseas, con el consiguiente riesgo de extensión a estructuras vecinas, lo que da lugar a complicaciones tanto intra- como extracraneales (las primeras son las más frecuentes). La sospecha clínica ante situaciones de riesgo puede orientar el diagnóstico y permitir la instauración de un tratamiento precoz para evitar la progresión de la infección con el desarrollo de complicaciones mayores.

Se presenta el caso de una niña de 4 años con diagnóstico de MA, que desarrolló sintomatología neurológica, motivo por el cual se amplió el estudio. Se confirmó, de esta forma, la extensión de la infección, con el diagnóstico de absceso epidural y la trombosis de los senos transverso y sigmoideo.

\section{CASO CLÍNICO}

Niña de 4 años sin antecedentes patológicos y correctamente vacunada, que presentó un cuadro febril de 4 días de evolución. Se diagnosticó faringoamigdalitis aguda, y se inició el tratamiento con amoxicilina a razón de $50 \mathrm{mg} / \mathrm{kg} /$ día.

Tras 4 días de antibioterapia, continuó febril y comenzó con la sensación de giro de objetos y lateralización de la marcha. En la exploración, se constató el tímpano izquierdo congestivo y 
maniobra de Romberg positiva. Ante la clínica descrita, se sospechó OMA complicada, por lo que se extrajo analítica, que mostró proteína $\mathrm{C}$ reactiva (PCR) de 30,6 mg/dl y 15720 leucocitos / mcl con 12500 neutrófilos / mcl.

FIgURA 1. Tomografía computada craneal con imagen ovoidea, hipodensa y bien delimitada que corresponde a un absceso epidural intracraneal

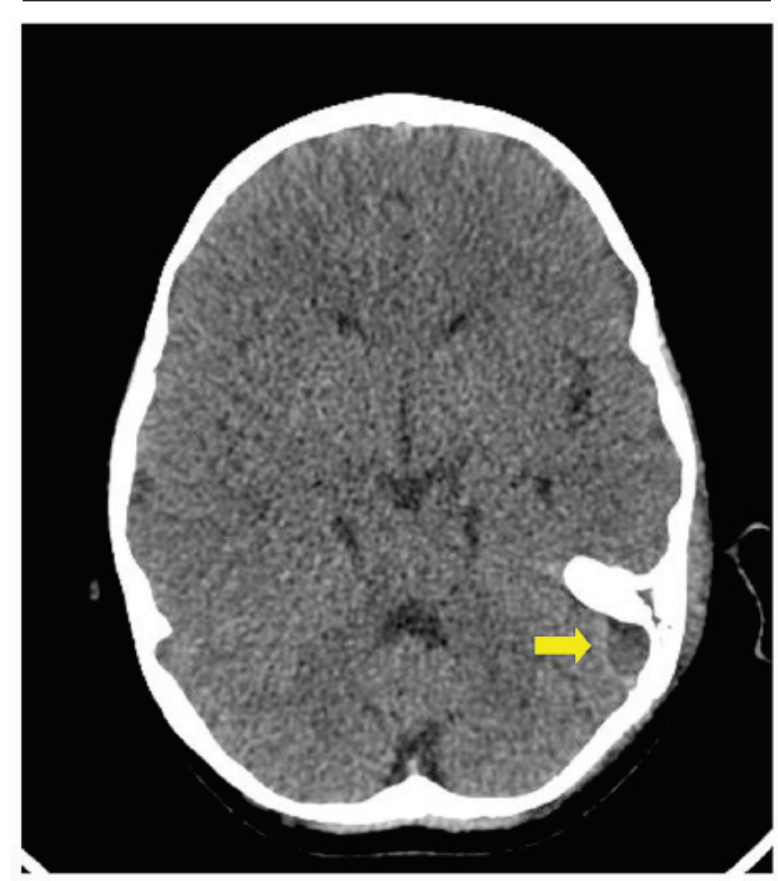

Se decidió el ingreso hospitalario para el tratamiento con amoxicilina-clavulánico intravenoso. Tras 24 horas, la paciente quedó afebril, pero se evidenció el despegamiento del pabellón auricular izquierdo, y se asoció cefalea a los síntomas periféricos previos. Por este motivo, se decidió la realización de la tomografía axial computada (TAC) craneal, y se visualizó una colección epidural izquierda de $7 \times 10 \times 24 \mathrm{~mm}$ (Figura 1).

Ante la MA con complicación intracraneal, se modificó la antibioterapia y comenzó con cefotaxima, vancomicina y metronidazol. Al mismo tiempo, se solicitó la evaluación por parte de Otorrinolaringología, quienes realizaron el drenaje de mastoides y colocaron un tubo de drenaje transtimpánico. Se cultivó la muestra del drenaje mastoideo, con crecimiento de Streptococcus pyogenes, sensible a los antibióticos habituales.

Continuando el estudio, con el fin de descartar complicaciones asociadas al absceso epidural, se decidió realizar una resonancia magnética nuclear (RMN) craneal. Se evidenció la trombosis de los senos transverso y sigmoideo (Figura 2), y se añadió enoxaparina subcutánea al tratamiento.

La evolución de la paciente fue favorable, con desaparición de la cefalea y la clínica periférica a las 48 horas de la cirugía y del cambio de antibiótico. Se mantuvo el tratamiento intravenoso durante 4 semanas y se continuó,

FIGURA 2. Resonancia magnética nuclear con contraste con defecto de repleción de la región distal del seno transverso y calibre filiforme del sigmoideo, sugestivos de trombosis venosa
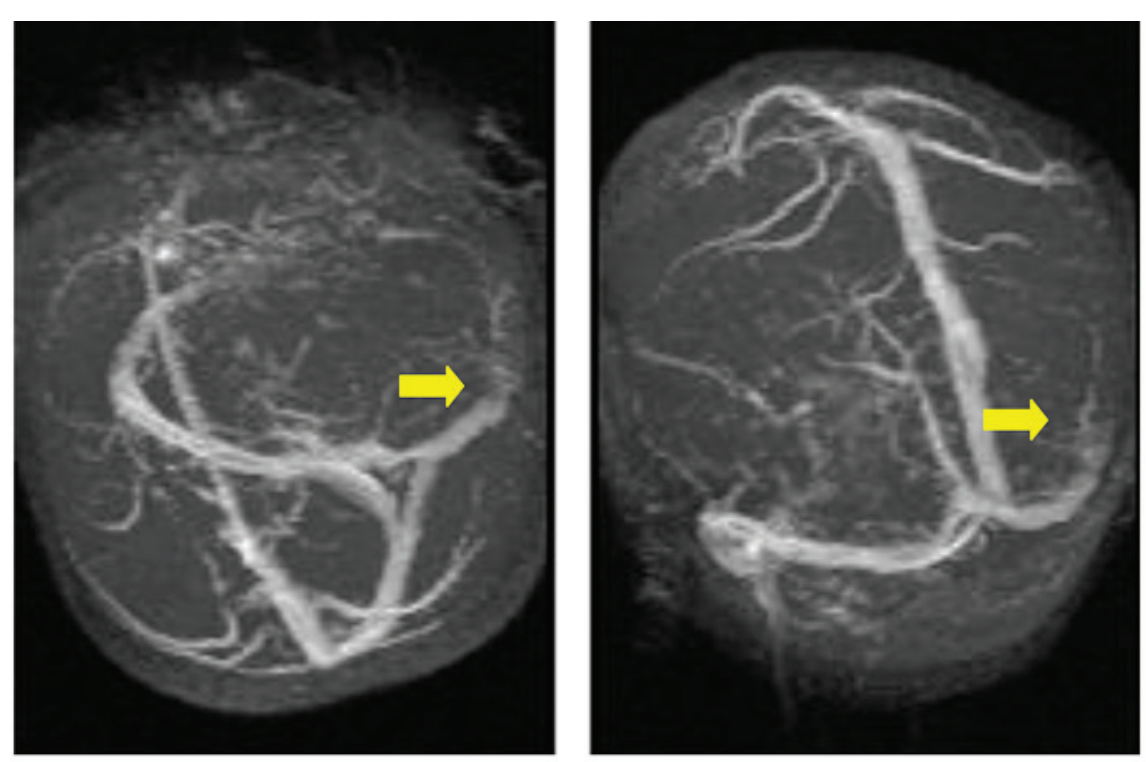
posteriormente, con linezolid oral en el domicilio por 7 semanas más. Se realizó un seguimiento estrecho, y se observó la normalización de la RMN 4 meses después del ingreso, momento en el cual se suspendió el tratamiento anticoagulante.

\section{DISCUSIÓN}

La MA es la complicación más frecuente de la OMA y se define como la infección de las celdillas mastoideas del hueso temporal. El diagnóstico es, fundamentalmente, clínico, con la presencia de signos inflamatorios locales, como el despegamiento del pabellón auricular, el eritema y el edema de la región mastoidea, junto con signos de OMA en la otoscopia.

En las últimas décadas, se ha documentado un aumento en la frecuencia de la MA en los niños. Las causas no están totalmente aclaradas, aunque hay diferentes teorías, como la resistencia antibiótica bacteriana, un tratamiento antibiótico inadecuado o un retraso en el tratamiento de las OMA. Ninguna de estas situaciones por sí mismas parecen ser las responsables del aumento en la incidencia de la MA, por lo que se cree que se trata de un proceso multifactorial. ${ }^{1}$

La administración de terapia antibiótica de forma rutinaria en las OMA no ha demostrado evitar la evolución de estas hacia la MA, ya que el $36-87 \%$ de los casos habían recibido terapia antibacteriana previa al diagnóstico de infección mastoidea. No obstante, la antibioterapia rutinaria en las OMA puede, en algunos pacientes, mejorar los signos de la otitis en la membrana timpánica, los cuales pueden no estar presentes en el momento de la aparición de la MA y dar lugar a un retraso en el diagnóstico. ${ }^{2}$ Los pacientes tratados con antibioterapia prolongada (1-2 semanas) previa al diagnóstico de complicación intracraneal pueden presentar menos signos y síntomas otológicos que los no tratados. $^{3}$

Una vez establecida, la infección puede avanzar hacia estructuras vecinas, por vía sanguínea o por contigüidad, y producir complicaciones intra- o extracraneales, las cuales aparecen en un 5-29\% de las mastoiditis. ${ }^{4}$ Dentro de la afectación intracraneal, se incluye la meningitis, el absceso del lóbulo temporal o cerebelo, el absceso epidural o subdural y la trombosis de seno venoso. Las posibles complicaciones extracraneales son el absceso subperióstico, la parálisis del nervio facial, la pérdida auditiva, la laberintitis, la osteomielitis y el absceso de Bezold. ${ }^{5}$
Una revisión sistemática publicada en el año 2009, en la que se analizaron los datos de pacientes con diagnóstico de MA, mostró que el $17 \%$ (el 2-100 \%) de los pacientes presentaron una complicación intracraneal. Las más frecuentes fueron la meningitis, la trombosis de seno venoso y los abscesos subdural y epidural. ${ }^{6}$

La revisión mencionada describió los porcentajes como medias de porcentajes (no se había recalculado sobre el total de pacientes), por ello, el $17 \%$ figuraba como media y tenía un rango tan amplio. En nuestro caso, la paciente presentó 2 de las complicaciones más frecuentes según esta revisión: absceso epidural y trombosis de senos venosos.

Los gérmenes implicados con más frecuencia en las MA son Streptococcus pneumoniae, Streptococcus pyogenes, Haemophilus influenzae y Moraxella catarrhalis. ${ }^{7}$ Las OMA causadas por Streptococcus pyogenes presentan un riesgo relativo mayor de evolucionar hacia MA que aquellas causadas por Streptococcus pneumoniae o Haemophilus influenzae (11,6/1000 frente a 2,2 y $0,3 / 1000$ pacientes). ${ }^{8}$

No existen unos criterios diagnósticos de MA establecidos. El diagnóstico se basa en la presencia de inflamación o eritema de la zona retroauricular, despegamiento del pabellón auricular y dolor en la zona afectada. Pese al porcentaje no desdeñable de complicaciones intracraneales, la realización de la prueba de imagen de rutina en los niños con MA es controvertida, y es de elección la TAC.

Algunos autores recomiendan individualizar la decisión de realizar una prueba de imagen según la presencia de signos o síntomas que puedan hacer sospechar una complicación: focalidad neurológica, afectación del estado general, no respuesta al tratamiento tras $48-72$ horas de antibioterapia intravenosa adecuada, larga duración de los síntomas o tratamiento antibiótico previo a la hospitalización. ${ }^{9,10}$ Sin embargo, otros autores recomiendan la realización de la prueba de imagen de forma rutinaria ante la MA, debido a la dificultad de determinar unos criterios que establezcan el riesgo de presentar una complicación. ${ }^{11}$ En nuestro caso, la presencia de clínica neurológica motivó la realización de la TAC craneal, y se observaron las complicaciones intracraneales descritas.

No existe un consenso claro en cuanto al tratamiento quirúrgico en la MA. El tratamiento inicial suele ser conservador y consiste en antibioterapia que cubre los gérmenes más 
habituales con o sin miringotomía. Será preciso evaluar la necesidad de una mastoidectomía en los casos en los que no se aprecie una mejoría en las primeras 24-48 horas de tratamiento conservador o cuando se sospeche alguna complicación. Una revisión sistemática muestra que, en los casos de MA no complicada, la actitud terapéutica adoptada con mayor frecuencia es la conservadora frente a la cirugía agresiva (el 93,2\% vs. el 6,8\%); mientras que los casos complicados con absceso subperióstico son tratados con cirugía agresiva con mayor frecuencia que con cirugía conservadora. ${ }^{4}$

El absceso epidural intracraneal en la edad pediátrica puede ser tratado de forma conservadora con tratamiento antibiótico de amplio espectro prolongado y drenaje del foco infeccioso, sin necesidad de realizar un procedimiento neuroquirúrgico. En cuanto a la trombosis de seno venoso, el tratamiento debe ir dirigido a recanalizar el seno y a evitar la progresión del trombo. En la edad pediátrica, se sugiere la anticoagulación con heparina de bajo peso molecular, aunque no existen estudios con suficiente evidencia para establecer una recomendación fuerte en cuanto al tratamiento de esta complicación. ${ }^{12}$ En el estudio de Ghadersohi et al., se incluyeron pacientes que presentaron MA asociada a alguna complicación intracraneal. En la mayoría, se realizó una cirugía otorrinolaringológica, y solo el $14,6 \%$ requirieron tratamiento neuroquirúrgico. La mayoría de pacientes afectos de absceso epidural y trombosis de senos venosos fueron tratados con mastoidectomía (el 93,8 \% y el $71,4 \%$, respectivamente). ${ }^{13}$ El pronóstico de estas complicaciones suele ser favorable si se realiza el diagnóstico precoz y el tratamiento adecuado..$^{14}$

\section{REFERENCIAS}

1. Bartolomé Benito M, Pérez Gorricho BP. Acute mastoiditis: increase in incidence and controversies in antibiotic treatment. Rev Esp Quimioter. 2006; 19(4):337-41.

2. American Academy of Pediatrics Subcommittee on management of acute otitis media. Diagnosis and management of acute otitis media. Pediatrics. 2004; 113(5):1451-65.

3. Osborn AJ, Blaser S, Papsin BC. Decisions regarding intracranial complications from acute mastoiditis in children. Curr Opin Otolaryngol Head Neck Surg. 2011; 19(6):478-85.

4. Loh R, Phua M, Shaw CL. Management of paediatric acute mastoiditis: systematic review. J Laryngol Otol. 2018; 132(2):96-104.

5. Wald E. Acute mastoiditis in children: clinical features and diagnosis. En UpToDate [en línea]. [Acceso: 31 de julio de 2019]. Disponible en: https://www.uptodate.com/ contents / acute-mastoiditis-in-children-clinical-featuresand-diagnosis?search=Acute $\% 20$ mastoiditis $\% 20 \mathrm{in} \% 20$ children: $\% 20$ Clinical $\% 20$ features $\% 20$ and $\% 20$ diagnosis\&source $=$ search_result\&selectedTitle $=1 \sim 150 \&$ usage_ type=default\&display_rank $=1$.

6. Van der Aardweg M, Rovers M, De Ru JA, Albers FW, et al. A systematic review of diagnostic criteria for acute mastoiditis in children. Otol Neurotol. 2008; 29(6):751-7.

7. Kordeluk S, Kraus M, Leibovitz E. Challenges in the management of acute mastoiditis in children. Curr Infect Dis Rep. 2015; 17(5):479.

8. Segal N, Givon-Lavi N, Leibovitz E, Yaqupsky P, et al. Acute otitis media caused by Streptococcus pyogenes in children. Clin Infect Dis. 2005; 41(1):35-41.

9. Chesney J, Black A, Choo D. What is the best practice for acute mastoiditis in children? Laryngoscope. 2014; 124(5):1057-8.

10. Oestreicher-Kedem Y, Raveh E, Kornreich L, Popovtzer A, et al. Complications of mastoiditis in children at the onset of a new millennium. Ann Otol Rhinol Laryngol. 2005; 114(2):147-52.

11. Luntz M, Bartal K, Brodsky A, Shihada R. Acute mastoiditis: the role of imaging for identifying intracranial complications. Laryngoscope. 2012; 122(12):2813-7.

12. Wong BY. Hickman S, Richards M, Jassar P, et al. Management of paediatric otogenic cerebral venous sinus thrombosis: a systematic review. Clin Otolaryngol. 2015; 40(6):704-14.

13. Ghadersohi S, Young NM, Smith-Bronstein V, Hoff S, et al. Management of acute complicated mastoiditis at an urban, tertiary care pediatric hospital. Laryngoscope. 2017; 127(10):2321-7.

14. Groth A, Enoksson F, Hultcrantz M, Stalfors J, et al. Acute mastoiditis in children aged 0-16 years-a national study of 678 cases in Sweden comparing different age groups. Int J Pediatr Otorhinolayngol. 2012; 76(10):1494-500. 